\title{
The Microanalytic Data Analysis Package
}

\author{
KIM KIENAPPLE \\ Mount Saint Vincent University, Halifax, Nova Scotia, Canada
}

\begin{abstract}
The Microanalytic Data Analysis Package is a series of BASIC programs designed to facilitate the analysis of observational data. The package provides: (1) an organizational framework for microanalytic data that is common to all programs; (2) reliability, frequency, cooccurrence, latency, distribution, and lag sequential analysis programs; and (3) data reduction programs.
\end{abstract}

Attempts to computerize the abstraction of summary statistics from data obtained through observational methods have ranged from programs designed to calculate specific measures (Dodd, Bakeman, Loeber, \& Wilson, 1981; Quera \& Estany, 1984; Sackett, Holm, Crowley, \& Henkins, 1979) to more general-purpose programming languages, such as CRESCAT (Kaye, 1979). Problems arise in the use of these programs because of the need to recode the data to match the input format or, in the case of CRESCAT, to learn a new (or first) programming language. The Microanalytic Data Analysis Package (MADAP) represents an attempt to improve upon these previous data reduction and analysis tools by providing a comprehensive system of data analysis and reduction programs that use an analysisindependent data format and prompt the user for the analysis parameters. Microanalysis, as defined by Kaye (1982) and as used by a number of researchers (Fogel, 1982, 1985; Fogel \& Hannan, 1985; Hannan, 1982; Kienapple, 1983), is an event-sampling observation method that involves the real-time coding of subject performance records using a data organizing scheme that extracts both experimenter-defined categories of subject activity and time of occurrence. With event recording technology permitting accurate time measurement to a period of $.01 \mathrm{sec}$ and increasing theoretical specificity, the data derived from such studies are often so detailed that attempts to manually create summary measures become inordinately time-consuming, expensive, and prone to error.

A related aspect of microanalysis identified by Kaye (1982) is that the sheer volume of data produced by this method sometimes masks the most consequential findings. Exploratory data analysis is sometimes the only means to discover relationships that exist among the data. Without the aid of appropriate data reduction software, such exploratory analyses would be impractical.

\section{THE DATA ORGANIZING SCHEME}

The data organizing scheme is based on the division of subject performance into modes and categories. Modes are conceptually distinct areas of subject activity, whereas

The author's mailing address is: Department of Child Study, Mount Saint Vincent University, Halifax, Nova Scotia B3M 2J6, Canada. categories are more finely defined activities within a mode. Any real-time data can be analyzed through the use of this data scheme and the accompanying programs. For example, in the coding of the activities of a human infant, the infant's eyes may be identified as a mode, whereas such behaviors as "gaze at mother," "gaze at self," and "eyes closed" constitute categories within this mode. Mode classifications may also represent different experimental conditions, subjects, or other hypothesisrelevant divisions. The use of a mode designator follows from the statistical requirement that data items within a mode be mutually exclusive.

The level at which the categories are coded is dependent on reliability, sufficient detail, theoretical relevance, and mutual exclusiveness. MADAP places no restriction on the number of categories. Indeed, two of the programs within MADAP are designed to permit category collapsing or cross-mode amalgamation.

The data derived from any real-time observation can be structured according to the time of onset value, mode designator, and category label format. For example, the data item "10.51, RH-GRASP" may signify that at $10.51 \mathrm{sec}$ into the session the subject's right hand was grasping someone or something. MADAP provides a data entry program that has a certain amount of flexibility in defining the time value but enforces the mode-behavior category format. Before the user can enter data, a time template is created that consists of any combination of place holders for hour, minute, second, or hundredths of a second. The template determines how the subsequent time values are to be interpreted since MADAP stores the time value in a second and a fraction of a second format. The mode designators can be up to 3 alphanumeric characters in length. The category designators can be up to 10 alphanumeric characters in length, although some programs truncate the category label to 4 characters in printed output. The use of alphanumerics aids in the interpretation of the output since the designators can be of strict mnemonic value. Each mode is entered separately, and the researcher has the option of combining all or some of the mode files into data files dependent on subsequently desired analysis. Currently, there is a limit of 15 modes in a merged file. Naming of the data files is under total control of the researcher; however, storage and organization of the data files is a function of the hardware and 
operating system. The data files are stored as text files, allowing access by programs outside of MADAP. MADAP does not contain a data file editing program; however, the storage format is accessible by any text editor. The MADAP program that performs the merging of the individual mode files sorts the data by time of occurrence. This organization provides a flexible format for further data reduction, analysis, and transcription of the data.

\section{RELIABILITY ANALYSIS}

Since reliability of the coded information is best determined through a convergence of methods that provide estimates of accuracy and consistency, MADAP contains two programs that provide commonly used measures of reliability.

The first calculates Cohen's kappa for real-time data and is based on a procedure outlined by Conger (1985). The calculation of kappa with continuous data is based on the argument that moment-by-moment agreement is superior to the arbitrary imposition of time intervals, since the latter procedure produces values for kappa that are dependent on the time interval selected. Chance agreement is defined as the probability that two observers will determine that a behavior category occurred at the same randomly selected time. Total chance agreement is the sum of the cross-products of the marginal proportions. The proportions are created by dividing the total duration of a category by the total observation time. Observed agreement is defined as those occasions when both observers assign the same behavior category. Total observed agreement is the sum of the proportion of within-category agreements (i.e., the proportions lying on the diagonal). The MADAP program KAPPA provides the kappa value as well as the option of printing the matrix upon which kappa is calculated. The matrix output is especially useful in enabling the researcher to identify behavior categories that are being confused by the coders.

The second program determines percent of agreement and Kaye's (1980) estimates of reliability. This program considers a single behavior category at a time. Percent of agreement is calculated according to Hollenbeck (1978), except that realignment of the time stream is not performed. Both kappa and the percent of agreement estimates provide an indication of the consistency achieved in the coding. Kaye's (1980) formula is based on the assumption that real-time coding consists of correctly coded events, missed events, and false alarms and thus, Kaye argued, can be regarded as an estimate of accuracy. This measure depends on the assumption that coders are equally reliable, errors are independent, and the numbers of missed events and false alarms are equal. Kraemer (1982) questioned the validity of Kaye's formula in light of the lack of a criterion judgment and questioned the use of the Poisson distribution in significance testing. In reply, Kaye and Sanathanan (1982) noted that Kraemer's criticisms resulted from an attempt to apply Kaye's (1980) model in situations for which the model was not designed and that, regardless of the significance testing criticism, the reliability estimate is still a valid procedure.

\section{DATA ANALYSIS}

MADAP contains five data analysis programs. In keeping with the notion that microanalysis is partly exploratory analysis, each of the analysis programs includes an operator that performs a "logical or" combining operation on the modes and categories. This "or" operator allows the user to create events or states that consist of one or more modes and categories. The actual use of this operator will be made apparent through a few examples. In data containing a category that represents a child gazing at a peer, the "or" operator can be used to create a new event called mutual attention. This is an example of the cross-mode use of the "or" operator, where individual children are represented by different mode designators. Within-mode use of the "or" operator can occur when the researcher discovers that currently existing detail in the data is too fine to permit readily interpretable results or when the reliability analysis indicates that two categories are being confused and a decision is made to collapse them into one category. The "or" operator permits extensive data manipulation without altering the original data set.

The EVENT analysis program provides frequency and duration measures for single behavior categories or events. The output consists of frequency, duration, rate per minute, and proportion measures. All of the data analysis programs write the summary measures to a disk file that can be submitted to any statistical package without re-entering the results. The COOCCURRENCE program provides commonly used estimates of cooccurrence and transitional probabilities. Both raw duration and percentage estimates of cooccurrence are provided. The LATENCY program provides summary measures similar to those of the event program, except that the measures are based on the lag between the onset of one behavior category or event and another. The LATENCY program is also capable of calculating these measures when an intervening behavior category or event is specified.

The fourth set of programs provides analyses related to the frequency distribution of durations of behavior categories or events. The first program in this series calculates the frequency distribution of user-determined duration intervals. A plot of frequency versus log time is provided. In the second program, a procedure outlined by Lilliefors (1969) is used to test the resultant frequency distribution against the exponential distribution using the Kolmogorov-Smirnov statistic. This distribution matching test is essentially a test of randomness for the frequency distribution of the intervals.

The last set of data analysis programs consists of concurrent and nonconcurrent lag sequential analysis programs (Quera \& Estany, 1984) adapted to the MADAP data scheme. These programs are based on Sackett et al.'s (1979) lag sequential analysis formula with Allison and 
Liker's (1982) modification to the $z$-score calculation. Interpretation of the output from these programs can be aided by referring to Sackett's (1980) guide to the use of lag sequential analysis.

\section{DATA REDUCTION}

The final set of programs in MADAP consists of data modification programs that allow the researcher to create modified data files. The first permits the collapsing of behavior categories, whereas the second creates new events or states that are the amalgamation of separate mode-behavior category combinations. These programs make permanent modifications to the original data files.

Given the predominance of high-capacity diskettes and hard-disk drives, MADAP has a feature that allows the user to process any number of data files through any of the data analysis or reduction programs with no operator intervention.

\section{AVAILABILITY}

MADAP is written in compiled BASIC and is available for both CP/M and MS-DOS computers. To obtain MADAP and a reference manual on disk, send two formatted disks in a returnable diskette mailer to the author.

\section{REFERENCES}

Allison, P., \& LiKer, J. (1982). Analyzing sequential categorical data on dyadic interaction: A comment on Gottman. Psychological Bulletin, 91, 393-403.

Conger, A. (1985). Kappa reliabilities for continuous behaviors and events. Educational \& Psychological Measurement, 45, 861-869.

Dodd, P., Bakeman, R., Loeber, R., \& Wilson, S. (1981). JOINT and SEQU: FORTRAN routines for the analysis of observational data. Behavior Research Methods \& Instrumentation, 13, 686-687.

FoGEL, A. (1982). Early adult-infant interaction: Expectable sequences of behavior. Journal of Pediatric Psychology, 7, 1-22.

Fogel, A. (1985). Coordinative structures in the development of ex- pressive behavior in early infancy. In G. Ziven (Ed.), The development of expressive behavior: Biology-environment interactions (pp. 249-267). New York: Academic Press.

Fogel, A., \& Hannan, T. E. (1985). Manual actions of nine- to fifteenweek-old human infants during face-to-face interaction with their mothers. Child Development, 56, 1271-1279.

Hannan, T. E. (1982). Young infants' hand and finger expressions: An analysis of category reliability. In T. M. Field \& A. Fogel (Eds.), Emotion and early interaction (pp. 253-265). Hillsdale, NJ: Erlbaum.

Hollenbeck, A. R. (1978). Problems of reliability in observational research. In G. P. Sackett (Ed.), Observing behavior (Vol. 2, pp. 79-98). Baltimore: University Park Press.

KAYE, K. (1979). CRESCAT: Software system for analysis of sequential or real-time data [Computer program manual]. Chicago: University of Chicago.

KAYE, K. (1980). Estimating false alarms and missed events from interobserver agreement: A rationale. Psychological Bulletin, 88, 458-468.

KAYE, K. (1982). The moral philosophy of microanalysis. In T. M. Field \& A. Fogel (Eds.), Emotion and early interaction (pp. 237-251). Hillsdale, NJ: Erlbaum.

Kaye, K., \& Sanathanan, L. (1982). Reply. Unpublished manuscript.

KIENAPPLE, K. (1983). From recognition to categorization: The development of conceptual knowledge in 7- and 10-month-old infants. Unpublished doctoral dissertation. Purdue University, Lafayette, IN.

Kraemer, H. (1982). Estimating false alarms and missed events from interobserver agreement: Comment on Kaye. Psychological Bulletin, 92, 749-754.

Lilliefors, H. (1969). On the Kolmogorov-Smirnov test for the exponential distribution with mean unknown. American Statistical Association Journal, 64, 387-389.

Quera, V., \& EstanY, E. (1984). ANSEC: A BASIC package for lag sequential analysis of observational data. Behavior Research Methods, Instruments, \& Computers, 16, 303-306.

SACKETT, G. (1980). Lag sequential analysis as a data reduction technique in social interaction research. In D. Sawin (Ed.), Exceptional infant 4: Psychosocial risks in infant-environment transactions (pp. 300-340). New York: Brunner/Mazel.

Sackett, G., Holm, R., Crowley, C., \& Henkins, A. (1979). A FORTRAN program for lag sequential analysis of contingency and cyclicity in behavioral interaction data. Behavior Research \& Instrumentation, 11, 366-378.

(Manuscript received August 29, 1986; revision accepted for publication January 30, 1987.) 